\title{
Un vaccin contre la leishmaniose
}

Les lymphocytes auxiliaires Th 1 jouent un rôle déterminant dans la défense de l'organisme contre les parasites intracellulaires. En réponse à une stimulation antigénique, ces cellules sécrètent des cytokines (IFN $\gamma$, TNF $\alpha$, IL2) qui activent et mobilisent d'autres types cellulaires comme les macrophages. Le rôle primordial des lymphocytes Thl est particulièrement bien établi dans le cas des infections par les leishmanies, des protozoaires flagellés qui parasitent les macrophages. Ces organismes sont transmis par les insectes et sont responsables des leishmanioses, un ensemble de maladies dont les symptômes cliniques sont variables selon les individus et les souches de parasites. L'étude des leishmanioses a été facilitée par l'existence de souris susceptibles à l'infection par ce parasite. Cet excellent modèle animal a, en particulier, permis de montrer que les lymphocytes Th1, mais non Th2, étaient indispensables à la guérison. Ce résultat a été confirmé par l'isolement de clones de lymphocytes $\mathrm{T}$ protecteurs, c'est-à-dire capables, lorsqu'ils sont inoculés à des souris, de les protéger contre une infection. $\mathrm{Si}$ tous les clones protecteurs isolés jusqu'à présent sont de type Thl, tous les clones Thl ne sont pas protecteurs, ce qui suggère qu'aussi bien le phénotype que la spécificité des lymphocytes $\mathrm{T}$ est déterminante. Outre leur intérêt d'un point de vue fondamental, l'isolement de ces clones constituait une première étape sur la voie de l'identification de protéines parasitaires susceptibles d'être utilisées pour la préparation de vaccins.

Malheureusement, identifier les anti- clones T protecteurs n'est pas chose facile. En effet, contrairement aux lymphocytes B qui reconnaissent des molécules solubles, les lymphocytes $\mathrm{T}$ réagissent avec des peptides antigéniques associés à des molécules de surface codés par le complexe majeur d'histocompatibilité (CMH) $[1,2]$. Ces peptides, issus de la dégradation de protéines présentes dans le milieu extracellulaire, sont présentés à la surface de cellules spécialisées (cellules présentatrices de l'antigène, CPAg) sous forme de complexes peptide/CMH. La reconnaissance de ces complexes par le lymphocyte $\mathrm{T}$ provoque son "activation" et la sécrétion de cytokines. Chercher à identifier la cible d'un lymphocyte $\mathrm{T}$ revient donc à rechercher une protéine dont la dégradation produit un peptide antigénique, qui une fois associé aux molécules de $\mathrm{CMH}$, est capable d'activer ce lymphocyte. Pour atteindre cet objectif, trois stratégies peuvent être envisagées. La première consiste à préparer un extrait brut contenant l'antigène recherché, par exemple un homogénat de parasites, et à fractionner les molécules qu'il contient en utilisant des méthodes de séparation physique. Les différentes fractions sont alors testées pour leur capacité de stimuler les lymphocytes $\mathrm{T}$ qui sont l'objet de l'étude. Si la protéine est abondante et si ses propriétés physiques permettent de l'obtenir sous une forme suffisamment pure, il est alors théoriquement possible de cloner son gène. Une deuxième stratégie envisageable consiste à incuber l'homogénat de parasites avec des CPAg. Ces cellules sont ensuite lysées en présence de détergent et les complexes peptides/CMH purifiés. Les peptides antigéniques peuvent alors être élués des molécules du CMH et leur séquence déterminée grâce à un spectrographe de masse couplé à une HPLC. Parmi tous les peptides obtenus, il reste alors à identifier celui qui est effectivement capable d'activer les lymphocytes $T$, une tâche particulièrement difficile si le peptide recherché est peu abondant. En fait, malgré les efforts de plusieurs laboratoires, aucune des deux approches précédentes n'a donné les résultats escomptés. C'est l'une des raisons pour laquelle la stratégie utilisée par l'équipe de Nicolas Glaichenhaus apparaît particulièrement séduisante [3].

En collaboration avec une équipe américaine, ces chercheurs de l'université de Nice-Sophia Antipolis ont tenté d'identifier l'antigène parasitaire avec lequel réagissait un des clones de lymphocytes Thl connu pour protéger d'une infection par le parasite Leishmania major. Pour cela, ils ont construit une banque d'ADNc à partir de la forme "promastigote" du parasite. Les clones bactériens constituant la banque ont été regroupés en lots de 5000 , et les protéines recombinantes produites et purifiées. Chaque lot de protéines a ensuite été incubé avec des CPAg et les lymphocytes $T$ du clone Thl. Parmi les cinquante lots analysés, un seul était capable de stimuler la sécrétion d'IFN $\gamma$ par les lymphocytes T. Cinq tours de criblage successifs ont été nécessaires à l'équipe de Glaichenhaus pour identifier un clone unique produisant une protéine capable d'activer les lymphocytes T. Cette protéine de $36 \mathrm{kDa}$, baptisée LACK (Leishmania homolog of the receptors of activated kinase (.), est présente chez. toutes les espèces de leishmanies, y compris celles qui sont responsables 
des leishmanioses viscérales, des formes particulièrement graves de ces maladies. Puisque I.ACK était capable de stimuler des lymphocytes $\mathrm{T}$ protecteurs, il restait à déterminer si son administration à des souris pouvait les protéger contre une infection par une dose létale de parasites. C'est effectivement le cas, en particulier lorsque cette protéine est mélangée à un adjuvant qui favorise l'émergence de lymphocytes Thl. Si LACK est un candidat prometteur pour la préparation d'un vaccin contre les leishmanioses, il reste à démontrer qu'un tel vaccin peut effectivement protéger des populations humaines. Les premiers essais cliniques devraient être pratiqués chez l'homme avant la fin de l'année. D'ores et déjà, une stratégie similaire peut être envisagée dans le cas des nombreuses autres maladies infectieuses dans lesquelles la mobilisation et l'activation des lymphocytes $\mathrm{T}$ auxiliaires est indispensable à la guérison.

N.G.
1. Ribourdin-combe (; Bertolino P', Calin-Laturens V, (ierlier D). Ia présentation de l'antigène aux lymphocytes $T$. médecine/sciences 1991; 7 : 67480.

2. Amigorena $S$. Transport intracellulaire des molécules de classe II du (:MH. mérlecine/sciences $1995 ; 11: 661-8$

3. Mougneau E, Altare F, Wakil AE, Zheng S, Coppola T, Wang Z, Waldmann R, Locksley R, (Blaichenhaus $\mathrm{N}$. Expression cloning of a Leishmania major protective T cell antigen. Science $1995 ; 268$ : 563-7.

\section{BRÈVES}

Thérapie génique du diabète : tous les espoirs sont-ils permis ? Ie diabète sucré de type I ou diabète insulinodépendant (DID) est une maladie d'étiologie complexe et mal connue, aboutissant à la destruction des cellules $\beta$ des îlots de Langerhans productrices d'insuline. Le traitement proposé actuellement repose sur des injections pluriquotidiennes d'insuline par voie souscutanée. Ce traitement est très contraignant et sa mauvaise observance expose à des complications aiguës parfois mortelles. En outre, même si un bon équilibre glycémique est maintenu, des complications dégénératives apparaissent à long terme. Il est donc clair que la prise en charge thérapeutique de ces patients peut être améliorée. La mise au point d'une thérapie génique symptomatique du DID est envisageable. C'est ce que montrent les travaux récents de l'équipe de Savio Woo [1]. Des rétrovirus recombinants porteurs de l'ADNc de la pro-insuline de rat sous le contrôle du LTR viral sont perfusés directement dans la veine porte des rats, 24 heures après une hépatectomie partielle. Cette technique permet de cibler presque exclusive- ment le foie où le pourcentage d'hépatocytes transduits est de $1 \%$ à $10 \%$; il est cent fois moindre dans la rate et nul dans les autres organes. Les ARN spécifiques du transgène ne sont détectables que dans le foie. Deux semaines plus tard, les rats sont traités par de fortes doses de streptozotocine, un antibiotique toxique qui entraîne une destruction de la quasi-totalité des cellules $\beta$ des îlots de Langerhans. Chez les rats témoins, l'insulinémie résiduelle est indétectable au bout de quatre jours. En revanche, on n'observe aucune modification de l'insuline sérique chez les rats qui ont reçu le gène d'intérêt, ce qui témoigne d'une production extra-pancréatique. Ia carence en insuline chez les rats témoins provoque une élévation du glucagon et l'apparition d'une acidocétose sévère qui entraîne le décès en quatre à six jours. L'activité de l'insuline est suffisante chez les rats traités pour prévenir ces complications aiguës. Cependant, la glycémie à l'état nourri est élevée et identique dans les deux groupes de rats. Au cours du jeûne, la glycémie se normalise plus rapidement chez les rats transduits et reste, après vingt-quatre heures de jeûne, audessus du seuil d'hypoglycémie. Les rats traités par les rétrovirus restent donc diabétiques, mais présentent une meilleure régulation de leur glycémie au cours du jeûne, sans apparition d'hypoglycémie. Ces travaux montrent que la thérapie génique du diabète n'est pas une utopie et que le foie est un organe de choix pour cibler le gène de l'insuline. Cependant, les conditions de cette étude ne sont pas optimales: le produit du transgène qui est sécrété est la pro-insuline dont l'activité est inférieure à $10 \%$ de celle de l'insuline mûre et la production de l'hormone n'est pas réglée en fonction de la glycémie puisque le transgène est sous le contrôle du L.TR viral. De plus, le suivi des rats traités ne dépasse pas vingt-et-un jours car ils décèdent audelà de trois semaines d'anomalies rénales secondaires à la toxicité de la streptozotocine, ce qui empêche donc l'étude de la maladie diabétique chronique et de sa prévention chez les animaux transduits.

[1. Koloda TM, el al. Proc Natl Acad Sci USA 1995; 92 : 3293-7.] 
Liaison spécifique de l'érythropoiétine dans le cerveau. L'érythropoiétine (Epo) est la cytokine qui règle l'essentiel de l'érythropoïèse [1], et son site majeur de production est situé dans le foie fotal puis le rein adulte. Sur les progéniteurs érythroïdes, sa liaison à son récepteur spécifique, Epo-R, stimule la production des globules rouges et supprime le programme d'apoptose de ces cellules. Mais l'Epo, ainsi que son récepteur, est produite aussi dans d'autres organes, en particulier par les cellules nerveuses: elle présente des propriétés neurotrophiques pour les cellules nerveuses fœtales et permet la survie de neurones endommagés in vivo chez le rat [2]. L'Epo ne franchissant pas la barrière hémo-méningée doit être synthétisée sur place, si elle joue un rôle physiologique dans le cerveau. L'ARNm de l'Epo a été effectivement mis en évidence dans le cerveau de rats hypoxiques; en outre, certaines tumeurs du système nerveux central chez l'homme sont associées à des polyglobulies et sécrètent l'Epo. Mais, la sécrétion d'Epo par les cellules nerveuses estelle réglée par l'hypoxie comme dans le rein, et où est-elle synthétisée? Digicaylioglu el al. (Zurich, Suisse) ont mesuré une augmentation de vingt fois de l'ARNm de l'Epo dans le cerveau de souris exposées à une atmosphère contenant $0,1 \%$ de monoxyde de carbone, procédé créant une hypoxie fonctionnelle [3]. Dans ces conditions, la concentration d'Epo est multipliée par quarante dans la circulation systémique, mais elle doit se distribuer dans les tissus hématopoïétiques alors que l'Epo cérébrale ne quitte pas son territoire et agit probablement de manière paracrine. Ils ont analysé par autoradiographie la liaison d'125I-Epo recombinante à des coupes de tissus nerveux. C'est dans la substance blanche que résident le plus grand nombre de sites de liaison de l'Epo, ce qui amène à penser que les axones pourraient porter les récep- teurs d'Epo. L'Epo joue-t-elle un rôle dans la neurogenèse? L'étude d'embryons de souris montrant l'expression d'Epo-R pourrait le suggérer. Masuda et al. (Japon) rapportaient récemment que des cellules cérébrales de foetus de rat en culture primaire, immortalisées par l'antigène T du SV40, accumulaient de l'Epo d'une manière dépendante de l'oxygène, et localisaient sa production aux astrocytes [4]. L.'Epo pourrait donc jouer un rôle physiologique dans la défense des cellules nerveuses contre l'hypoxie: on sait que, lors de l'hypoxie, la conductivité pour $\mathrm{K}^{+}$, $\mathrm{Na}^{+}$et $\mathrm{Ca}^{2+}$ des cellules nerveuses change et que le besoin cellulaire en ATP diminue, ce qui assure une certaine protection de ces cellules. L.'Epo pourrait provoquer des modifications membranaires; elle ne serait donc pas un facteur de croissance cantonné aux cellules hématopoïétiques, mais serait plurifonctionnelle à l'image des nombreux facteurs de l'hématopoïèse qui se lient aux récepteurs de la superfamille des cytokines.

[1. Lacombe C, Mayeux P. médecine/sciences 1995; 11 : 947-55.]

[2. Konishi Y, el al. Brain Res 1993; 609: 29-35.]

[3. Digicaylioglu M, el al. Proc Natl Acad Sci USA 1995; 92 : 3717-20.]

[4. Masuda S, el al. J Biol Chem 1994 ; 269: 19488-93.]

Des vecteurs rétroviraux ciblés pour la thérapie génique. L'administration in vivo de vecteurs de thérapie génique nécessite que ceux-ci puissent reconnaître leur cible et y transférer spécifiquement le gène thérapeutique. Depuis plusieurs années, de nombreuses équipes tentent ainsi de cibler des vecteurs non viraux ou viraux grâce à l'adjonction de ligands reconnus par des récepteurs présents à la surface des cellules cibles. Cependant, le succès avait été jusqu'ici très modeste. C'est la raison pour laquelle il faut saluer deux articles récemment publiés démontrant que l'introduction d'une séquenceligand dans la protéine d'enveloppe de rétrovirus leur permet effectivement d'infecter spécifiquement leur cellule cible. Kasahara el al., de l'équipe de Kan (San Francisco, CA, USA), viennent ainsi de produire des rétrovirus écotropes murins possédant une glycoprotéine d'enveloppe hybride avec remplacement de 150 acides aminés dans la région $\mathrm{N}$-terminale par la séquence de l'érythropoïétine [1]. Cela a été obtenu en créant une cellule d'encapsidation ayant intégré le gène hybride EPO-env. Ces cellules ont été ensuite infectées à l'aide d'un rétrovirus écotrope recombinant véhiculant les gènes neo (de résistance à l'antibiotique G418) et $\beta$-galactosidase. Des clones résistants au G418 et produisant des particules rétrovirales recombinantes infectieuses ont été alors isolés. Ces rétrovirus peuvent posséder deux types de protéines d'enveloppe, la glycoprotéine sauvage et la glycoprotéine hybride. Alors que des rétrovirus écotropes sont normalement complètement incapables d'infecter autre chose que des cellules murines, les vecteurs obtenus ici sont efficaces pour infecter des cellules humaines synthétisant le récepteur de l'érythroproïétine. L'efficacité pour des cellules murines est également très augmentée lorsque celles-ci ont préalablement été transfectées avec un vecteur d'expression pour le récepteur de l'érythropoïétine. Dans un autre article, Valsesia-Wittmann el al. décrivent l'infection de cellules de mammifère par des rétrovirus aviaires possédant une glycoprotéine d'enveloppe à laquelle a été intégrée une séquence d'une protéine d'adhérence, une intégrine [2]. Naturellement, ces résultats sont préliminaires, mais ils sont encouragants car ils représentent les premiers succès de ce type.

[1. Kasahara N, el al. Science 1994 ; $266: 1373-6$.

[2. Valesia-Wittmann C, el al. J Virol 1994 ; 68 : 4609-18.] 
Le système immunitaire manipulé corrige ses réactions autoimmunes. Les lymphocytes T CD4 se différencient en deux sous-populations (Thl et Th2) sous l'influence de stimulations antigéniques. Les deux types cellulaires produisent des cytokines antagonistes et se règlent mutuellement $\left(m / s n^{\circ} 5\right.$, vol. 10, p. 610). La différenciation des cellules précurseurs en cellules de type Th1 ou Th2 doit être parfaitement contrôlée et peut se traduire par une susceptibilité ou une résistance naturelle accrue à certaines infections ou réactions autoimmunes. Dans le cas de l'EAE (experimental allergic encephalomyeli(is), l'amélioration de la maladie est corrélée à un accroissement du nombre de lymphocytes Th2 et de leurs cytokines dans le cerveau. Cela suggère que les cellules Th2 pourraient freiner le développement de certaines maladies auto-immunes liées à une activation indésirable des cellules Thl. Après reconnaissance d'un antigène, les lymphocytes $\mathrm{T}$ sont activés s'ils reçoivent un signal de costimulation transmis par un système de récepteurs/ligands dont le plus connu est illustré par l'interaction des molécules B7.1 et B7.2 des cellules présentatrices avec les molécules CD28 et CTLA-4 des lymphocytes $\mathrm{T}[1,2]$. L'influence respective de $B 7.1$ et $B 7.2$ sur le développement des populations Thl et Th2 et sur l'évolution de l'EAE a été étudiée in vitro et in vivo à l'aide d'anticorps monoclonaux. Les anticorps anti-B7.1 diminuent l'incidence de la maladie et favorisent la production de clones Th2 dont le transfert à d'autres animaux est préventif et curatif. L'injection d'anticorps anti-B7.1 et anti-IL4 (une cytokine sécrétée par les lymphocytes Th2) n'a pas d'effet positif sur l'évolution de la maladie. L'injection d'anticorps anti-B7.2 provoque une aggravation de la maladie. Cela montre qu'il est possible, avec des anticorps anti-B7, de modifier le développement d'une maladie auto-immune en créant un déséquilibre Th1/Th2 protecteur.
Les retombées thérapeutiques de cette observation pourraient n'être pas négligeables dans les cas de maladies auto-immunes dont la cible est identifiée.

[1. Butor $\mathrm{C}$, et al. médecine/sciences $1995 ; 11: 703-11$.

[2. Richard Y, Galanaud P. médecine/sciences $1995 ; 11$ : 5 : 691-702.]

[3. Kuchroo V, et al. Cell $1995 ; 80$ : 707-18.]

Aquaporine-2 (AQP-2) : de la vésicule à la membrane apicale. Jo et al. (Boston, MA, et Madison, WI, USA) ont étudié le transfert de l'AQP-2 de la vésicule intracytoplasmique à la membrane apicale du canal collecteur où elle s'insère pour permettre l'entrée de l'eau dans la cellule $\left(\mathrm{m} / \mathrm{s} n^{\circ} 2\right.$, vol. 11 , p. 299). Ils ont utilisé des anticorps dirigés contre la synaptobrévine $\beta$, dénommée également VAMP-2 pour vesicle-associated membrane protein 2. Cette protéine est l'intermédiaire de l'exocytose de la vésicule synaptique dans le cerveau, et est probablement aussi l'un des composants responsables de la fusion de vésicules intracellulaires dans toutes les cellules $\left(\mathrm{m} / \mathrm{s} n^{\circ} 6-7\right.$, vol. 9, p. 802). La papille et la médullaire interne de rein de rat contiennent en abondance de la VAMP-2, mais seulement dans les endosomes les plus légers. La fusion de ces endosomes est inhibée in vitro par une préincubation avec des anticorps antiVAMP-2 ou avec la toxine tétanique (qui dégrade la synaptobrévine). Ainsi, la synaptobrévine serait impliquée dans la fusion de certains endosomes du canal collecteur rénal et pourrait être impliquée dans le "routage" de l'AQP-2 vers la membrane apicale (comme cela a été suggéré également pour le transporteur du glucose sensible à l'insuline) [1]. Deen et al. (Nimègue, Pays-Bas) ont utilisé une approche différente. Ils ont étudié trois mutants faux-sens de l'AQP-2, responsables de diabète insipide néphrogénique; ces protéines mutantes exprimées dans les ovocytes de xénope n'augmentent pas la perméabilité osmotique à l'eau. Les ARNm sont traduits avec la même efficacité pour les mutants et pour l'AQP-2 normale. Deux protéines mutantes sur trois sont moins stables que l'AQP-2 naturelle. Enfin, par immunotransfert et en immunochimie, seule l'AQP-2 normale est exprimée sur la membrane plasmique alors qu'un marquage intracellulaire est mis en évidence dans les ovocytes injectés avec les ARNc mutants. Ainsi, l'incapacité des AQP-2 mutantes de faciliter le transport d'eau est due au routage altéré de la protéine à la membrane [2] . [1. Jo I, et al. Proc Natl Acad Sci USA 1995 ; 92 : 1876-80.]

[2. Deen PMT, et al. I Clin Invest $1995 ; 95: 2291-6$.

Mimétisme moléculaire dans la sclérose en plaques. L'hypothèse du mimétisme moléculaire s'appuie sur l'existence d'épitopes croisés entre peptides viraux ou bactériens et peptides du soi. Il existe en effet un certain degré de dégénérescence dans la reconnaissance des peptides par les lymphocytes $T$ du fait même des mécanismes de sélection du répertoire $\mathrm{T}$ dans le thymus et de la variabilité antigénique des agents pathogènes. Les mécanismes conduisant à l'expansion de clones autoréactifs dans les maladies autoimmunes pourraient donc impliquer la reconnaissance d'épitopes étrangers ayant des similitudes structurales avec les épitopes du soi (épitopes de la MBP [protéine basique de la myéline], ou dans la sclérose en plaques). Dans ce cas, les virus causant des infections latentes ou persistantes pourraient être la source de tels épitopes croisés et provoquer une stimulation antigénique chronique de clones autoréactifs [1]. A partir des données structurales imposées par le récepteur d'antigène et le complexe majeur d'histocompatibilité pour la reconnaissance d'un pepti- 


\section{BRĖVES}

de immunodominant de la MBP, 129 peptides ont été sélectionnés à partir d'une banque et testés sur des clones autoréactifs anti-MBP de patients atteints de sclérose en plaques [2]. Sept peptides viraux et un peptide bactérien activent trois des clones testés mais un seul peptide (d'un papillomavirus) présente un mimétisme moléculaire important par alignement de séquences. Il ressort de cette étude qu'un groupe de virus (Herpes, influenza, papilloma) pourrait être impliqué dans un processus auto-immun. Cela explique pourquoi il est si difficile de relier la sclérose en plaques à un agent pathogène unique. L'élimination d'éventuels épitopes croisés des vaccins actuels et futurs réduirait peut-être la fréquence des accidents immunologiques postvaccinaux.

[1. Talbot P. médecine/sciences 1995 ; $11: 837-43$.

[2. Wucherfennig KW. Cell 1995 ; $80: 695-705$.

SIV nef-est pathogène chez les nouveau-nés. L'infection des macaques par le virus de l'immunodéficience SIV est le modèle le plus proche du SIDA de l'homme. SIV devient non pathogène lorsque son gène nef est délété : les macaques adultes infectés par ce virus mutant ne développent pas la maladie et sont protégés contre le virus sauvage $\left(m / s n^{\circ} 3\right.$, vol. $\left.11, p .478\right)$. La production d'un vaccin contre le SIDA à partir d'une souche virale nef- a donc été envisagée. Une fois de plus il faudra reconsidérer cet objectif car on sait maintenant que ce type de virus est en fait très pathogène pour le macaque nouveau-né [1]. Les constructions virales restent néanmoins très utiles pour étudier la fonction toujours mystérieuse de $n e \int$ et la réponse antivirale si différente entre les adultes et les nouveau-nés.

[1. Baba TW. Science 1995; 267 : 1820-5.] $\square$ L'hépatite B, cause aggravante du paludisme en Afrique? Le paludisme à $P$. falciparum représente en Afrique subsaharienne la cause principale de mortalité infantile. Si presque tous les enfants sont infestés par le parasite, les formes sévères, potentiellement mortelles - atteinte cérébrale ou anémie dramatique - représentent environ $1 \%$ des cas, soit un à deux millions de décès annuels chez de jeunes malades. L'équipe anglaise de Hill, qui poursuit sa recherche en Gambie, avait déjà noté le caractère apparemment protecteur de certains antigènes HLA $\left(m / s n^{\circ} 9\right.$, vol. 7, p. 981). Les facteurs prédisposants au paludisme sévère ne sont pas connus. Dans un récent travail, le groupe de Hill a examiné si l'antigène de l'hépatite $B$ pouvait être en cause [1]. En effet, le virus de l'hépatite B (HBV) est endémique en Afrique de l'Ouest et les chances sont grandes d'une infection simultanée au paludisme. On considère qu'environ $15 \%$ des enfants atteints restent porteurs du virus, et qu'une proportion encore supérieure exprime l'antigène de surface (HBsAg) pendant des années, souvent jusqu'à l'adolescence. L'antigène HBsAg a été recherché chez 1268 enfants, divisés en trois groupes, selon qu'ils présentaient un paludisme grave, un paludisme bénin, ou qu'ils n'étaient pas infestés. La présence de l'antigène HBsAg est très significativement plus élevée dans le premier groupe et semble indépendante des risques d'infection virale. Aucun argument ne vient étayer l'hypothèse d'une réactivation de l'expression de HBsAg par le paludisme, ni celle d'un tiers facteur créant une prédisposition commune aux deux infections. L'explication la plus probable rẻste que l'HBV influerait sur la progression du paludisme. Au cours d'une hépatite persistante, la majorité des hépatocytes sont infectés, ce qui réduit drastiquement l'expression des molécules HLA de classe I à leur surface. Les lymphocytes cytotoxiques $\mathrm{T}$ sont alors entravés dans leur rôle protecteur, qui est la lyse des cellules infectées par le parasite. Si l'infection virale prédispose au paludisme sévère, on peut envisager d'utiliser la vaccination HBV dans une prophylaxie du paludisme.

[1. Thursz MR, et al. Nature Med $1995 ; 1: 374-5$.]

Des arguments en défaveur de l'intervention des protéines antiapoptotiques Bcl-2 et Bcl-xL au niveau des espèces réactives de l'oxygène. médecine/sciences s'est fait l'écho du faisceau d'arguments indiquant que $\mathrm{Bcl}-2$ pourrait s'opposer aux espèces réactives de l'oxygène inducteur d'apoptose $\left(m / s n^{\circ} 2\right.$, vol. 10, p. 208). Deux articles, l'un d'une équipe japonaise d'Osaka [1] et l'autre d'une équipe anglaise de Londres [2] indiquent maintenant que les effets anti-apoptotiques de Bcl-2 [1,2] et de Bcl-xL [1] peuvent être observés pratiquement en l'absence d'oxygène. Shimizu et al. soumettent des cellules d'hépatome et de phéochromocytome de rat en culture à une hypoxie profonde provoquant l'apoptose cellulaire. La survie des cellules anoxiques en culture est prolongée par la surexpression de Bcl-2 ou de Bcl-xL [1]. Jacobson et Raff ont, quant à eux, déterminé l'effet antiapoptotique de $\mathrm{Bcl}-2$ dans des cellules cultivées à très faible concentration partielle en oxygène, dans des conditions où la formation d'espèces réactives de l'oxygène est très improbable. D'ailleurs, dans ces conditions, différents anti-oxydants n'ont aucun effet protecteur contre des inducteurs d'apoptose tels que la staurosporine, un inhibiteur de protéine kinase à large spectre. Comment expliquer la contradiction entre ce résultat et ceux antérieurement publiés? Bcl-2 et ses homologues pourraient agir, comme l'a récemment rappelé Martinou [3], en s'opposant à l'effet de protéases activées par le calcium. Dans ce cas, les espèces réactives de l'oxygène pourraient simplement 
être l'un des inducteurs possibles de cette augmentation du calcium intracellulaire inducteur d'apoptose, expliquant l'effet protecteur des antioxydants dans certains modèles d'apoptose en conditions aérobies. [1. Shimizu S, el al. Nature 1995 ; 374: 811-3.]

[2. Jacobson MD, Raff MC. Nature $1995 ; 374: 814-6$.]

[3. Martinou J. médecine/sciences $1995 ; 11$ : 367-73.]

Le signal apoptotique issu de Fas passe par la protéase ICE. Golstein [1] et Martinou [2] ont récemment exposé dans médecine/ sciences ce qui était connu des mécanismes de l'apoptose induite par la stimulation du récepteur membranaire Fas. Rappelons que cette protéine appartient à la même famille que les récepteurs du TNF (tumor necrosis factor) et peut être stimulée, physiologiquement, par une molécule dénommée Fas-ligand qui est présente à la surface des lymphocytes $\mathrm{T}$ cytotoxiques activés. La stimulation de Fas est ainsi l'un des mécanismes essentiels de la cytotoxicité associée à l'immunité cellulaire [1]. L'apoptose induite par une stimulation de Fas est un phénomène rapide, qui implique manifestement une cascade d'événements post-traductionnels car elle n'est bloquée, ni par les inhibiteurs de la synthèse protéique, ni par ceux de la transcription. Cette apoptose peut, en revanche, être bloquée par la protéine $\mathrm{Bcl}-2$, équivalent du produit du gène ced-9 du nématode Caenorhabditis elegans. Dans cet organisme, l'apoptose exige également l'action du gène ced-3 qui code pour une protéase équivalente de la protéine ICE chez les mammifères (interleukine-1 $\beta$-converting enzyme) [3]. Certains virus ont acquis au cours de l'évolution le moyen de retarder l'apoptose des cellules, ce qui leur permet de se multiplier plus longtemps dans une cellule vivante. C'est ainsi que les poxvirus bovins possèdent un gène,
$\operatorname{crmA}$, qui commande la synthèse d'un inhibiteur de la protéase ICE. Trois équipes, l'une japonaise d'Osaka, l'autre germano-belge de Heidelberg et de Gand, la troisième américaine du Michigan [4-6] viennent de démontrer que l'apoptose induite par l'activation de Fas, et aussi par la liaison du TNF à son récepteur, passait également par la protéase ICE : elle est inhibée par une surexpression du produit du gène $\mathrm{crmA}$, par un tétrapeptide inhibiteur de la protéase ICE et par une construction anti-sens antagoniste de l'ARN messager d'ICE. Certains phénomènes pathologiques (hépatite fulminante, maladies autoimmunes, SIDA...) comportent très probablement, dans leur mécanisme, l'induction d'une apoptose anormale. L'avenir dira si des inhibiteurs de l'apoptose, agissant par exemple au niveau de la protéase ICE, peuvent avoir un effet thérapeutique intéressant.

[1. Golstein P. médecine/sciences 1995; 11 : 99-104.]

[2. Martinou JC. médecine/sciences $1995 ; 11: 367-73$.]

[3. Labouesse M. médecine/sciences $1994 ; 10$ : 337-41.]

[4. Enari M, et al. Nature 1995 ; 375 : 78-81.]

[5. Los M, et al. Nature 1995 ; 375 : 81-3.]

[6. Tewari M, Dixit VM. J Biol Chem $1995 ; 270: 3255-60$.

Activation de la réponse des lymphocytes B par des dinucléotides CG déméthylés. Chez les vertébrés, les dinucléotides CpG - cette notation permet de différencier les deux nucléotides adjacents d'un brin, séparés par un pont phosphodiester, des paires appariées C:G sont sous-représentés car leur méthylation facilite la désamination des méthylcytosines en uraciles, secondairement transformées en thymines [1]. Les CpG restants sont, par ailleurs, eux-mêmes méthylés. En revanche, les systèmes de méthylation bactériens ne convertissent

pas les cytosines de ces dinucléotides CpG en 5 méthylcytosine. Krieg et al., de Iowa City (IO, USA) et Bethesda (MD, USA)[2] proposent que cette richesse de l'ADN bactérien en motifs $\mathrm{CpG}$ déméthylés explique leur pouvoir activateur des lymphocytes B. Cette activation peut coopérer avec une stimulation antigénique spécifique pour augmenter la synthèse d'anticorps. Des résultats similaires sont obtenus avec des oligonucléotides de synthèse riches en motifs $\mathrm{CpG}$ déméthylés. L'effet costimulateur de l'ADN riche en $\mathrm{CpG}$ déméthylé est indépendant de la coopération avec des lymphocytes $\mathrm{T}$ et son mode d'action n'est pas connu aujourd'hui. Ces résultats amènent, néanmoins, deux commentaires, l'un d'ordre évolutif, l'autre relié à l'utilisation thérapeutique des oligonucléotides antisens [3]. L'augmentation de la réponse lymphocytaire B à des infections bactériennes induite par le pouvoir costimulateur de l'ADN bactérien a pu être sélectionnée au cours de l'évolution comme un moyen d'améliorer la défense contre les maladies bactériennes. Par ailleurs, certains des effets des oligonucléotides antisens, qui ne semblent pas reliés à leur spécificité de séquence, pourraient être en rapport avec leur teneur en motifs CpG déméthylés. De tels oligonucléotides, quelles que soient leurs cibles théoriques, pourraient, en effet, agir comme des stimulateurs non spécifiques de l'immunité.

[1. Jordan B. médecine/sciences 1991; 7: 153-60.]

[2. Krieg AM, el al. Nature 1995 ; 374: 546-9.]

[3. Hélène C, Saison-Behmoaras E médecine/sciences 1994 ; 10 : 253-73.]

Polyphagie d'origine neurolo-
gique chez une souris déficiente en
récepteurs sérotoninergiques. La
sérotonine (5-hydroxytryptamine)
est un neurotransmetteur qui possè-
de de multiples récepteurs (quator-
ze sont connus aujourd'hui) dont la




\section{BRÈVES}

fonction spécifique reste incertaine. C'est la raison pour laquelle plusieurs équipes ont entrepris l'étude de cette fonction chez la souris par l'invalidation des gènes correspondants grâce à la technique de la recombinaison homologue. En septembre 1994, Saudou et al. (Strasbourg, France) montraient que des souris déficientes en récepteur 5$\mathrm{HT}_{\mathrm{IB}}$ étaient caractérisées par un comportement anormalement agressif vis-à-vis de leurs congénères [1]. Tecott el al. (San Francisco, CA, USA)[1] ont maintenant obtenu des souris déficientes en récepteurs de type $5-\mathrm{HT}_{2 \mathrm{C}}$. Ces souris, viables, semblent anatomiquement normales. Elles présentent, néanmoins, deux anomalies importantes : une obésité liée à une hyperphagie, et des convulsions, parfois mortelles. Par conséquent, un signal sérotoninergique passant par ce récepteur 5-HT 2c: semble jouer un rôle de modulation négative de l'appétit et fournir un tonus inhibiteur protégeant des crises convulsives. Ces résultats sont en accord avec certains des effets du médicament à la mode, le $\operatorname{Prozac}^{\circledast}$, qui augmente la transmission sérotoninergique par blocage de la recapture de la sérotonine par les terminaisons axonales : le Prozac $^{\circledR}$, utilisé comme antidépresseur, est actif dans certaines obésités par hyperphagie.

[1. Saudou, el al. Science 1994; 265 : 1875-8.]

[2. Tecott I.H, el al. Nature 1995 ; 374: 542-6.]

\section{Les promesses d'une hydrola-} se du PAF. Le PAF (platelet-activating factor) est un médiateur phospholipidique de très nombreux processus : inflammation, asthme, parturition, neurotransmission, etc. [1]. Il existe deux enzymes hydrolysant le PAF, l'une est intracellulaire et l'autre extracellulaire. Une des sous-unités de l'enzyme intracellulaire est commue des lecteurs de médecine/sciences puisque son gène est celui de la lissencéphalie, ou syndrome de Miller-Dieker $\left(m / s n^{\circ} 10\right.$, vol. 9, p. 1124 et $n^{\circ}$ 10, vol. 10, p. 1059). L'autre hydrolase est extracellulaire et son gène vient d'être cloné par une équipe comportant des chercheurs de Washington (DC) et de Salt Lake City (UT, USA) [2]. Cette équipe a ainsi pu préparer de la PAF-acétyl hydrolase recombinante dont l'administration a été testée dans différentes réactions pathologiques à l'injection de PAF chez l'animal. En injection locale ou intraveineuse, la PAF-acétyl hydrolase s'est révélée très active pour bloquer les effets pro-inflammatoires du PAF. En pathologie humaine, on sait que le PAF intervient dans de nombreux phénomènes inflammatoires et le choc septique. Un déficit en PAF-acétyl hydrolase a ainsi été observé chez des malades souffrant d'asthme, de lupus érythémateux disséminé et de choc septique, si bien que la compensation de ce déficit par l'injection de protéine recombinante pourrait avoir une réelle efficacité thérapeutique. Certaines susceptibilités aux accouchements prématurés pourraient également être secondaires à une insuffisance de l'hydrolyse du PAF circulant par la PAF-acétyl hydrolase et être ainsi améliorées par l'administration de l'enzyme. Des études ultérieures diront si cette enzyme hydrolysant le PAF est appelée à trouver une place thérapeutique plus convaincante que les inhibiteurs chimiques de PAF testés par différents laboratoires [3].

[1. Benvéniste J, Marsac J. médecine/sciences 1987 ; 3 : 506-7.]

[2. Pjoelker LW, el al. Nature 1995 ; 374 : 549-53.]

[3. Braquet $\mathrm{P}$, et al. médecine/sciences $1987 ; 3$ : 515-21.]
Des mécanismes de résistance à la carence en androgènes dans le cancer de la prostate : l'amplification et la mutation du gène du récepteur des androgènes. Des cellules, in vivo, ou en culture, résistent souvent à des agents cytolytiques grâce à une amplification et à une surexpression d'un gène de résistance (cible du produit cytolytique ou agent de détoxification) [1]. Un phénomène du même type vient d'être observé par des chercheurs finlandais d'Helsinki : dans $30 \%$ de 23 cancers de la prostate en rechute étudiés, Visakorpit et al. ont observé une amplification du gène codant pour le récepteur des androgènes [2]. Une telle amplification n'était jamais notée dans des échantillons prélevés avant le début d'un traitement comportant une orchidectomie et, une fois, l'administration d'œstrogènes. Il est probable que la synthèse augmentée du récepteur des androgènes permette une persistance de la croissance tumorale de cette tumeur hormonodépendante à très faible concentration d'androgène. Dans certains cas, la croissance tumorale en l'absence d'androgènes pourrait être expliquée par un autre mécanisme, d'ailleurs éventuellement associé à l'amplification : la mutation du récepteur, notée dans cinq observations sur dix de métastases survenant chez des malades castrés et traités (Taplin et al., Boston, MA, USA) [3]. Toutes les mutations sont localisées dans le domaine de liaison de l'hormone dont la spécificité est modifiée ; ainsi, les récepteurs mutants deviennent capables de fixer les œstrogènes et (ou) de la progestérone, et d'être activés par les hormones stéroïdes qui stimuleront ainsi la croissance tumorale en l'absence d'androgènes. Dans certains cas, la mutation peut transformer les anti-androgènes utilisés pour le traitement, par exemple le flutamide, en agonistes : un arrêt de ce traitement peut alors entraîner une régression tumorale. Les altérations observées du récepteur des androgènes ne sont pas notées 


\section{BRÈVES}

au niveau de la tumeur primitive initiale, ce qui signifie, soit qu'elles sont apparues au cours du traitement, soit qu'elles existaient au départ dans un très petit pourcentage de cellules qui ont ensuite été sélectionnées par le traitement.

[1. Szepetowski P, el al. médecine/science 1991 ; 7 : 14-21.]

[2. Visakorpit, el al. Nature (jenet $1995 ; 9$ : 401-6.]

[3. Taplin L, el al. $N$ lingl / Med $1995 ; 332$ : 1393-8]

VIH-Tat induit l'apoptose des lymphocytes non inf ectés. L'infection par le VIH provoque la disparition progressive des lymphocytes $T$ CD4 et un affaiblissement des déf enses immunitaires. La diminution du nombre de lymphocytes $\mathrm{T}$ reste inexpliquée et l'idée que le virus lui-même favorise le processus d'apoptose reste controversée, d'autant plus qu'il faudrait admettre que le signal d'apoptose engendré par le virus serait transmissible aux cellules non infectées. Il vient d'être montré que le transactivateur Tat du VIH [1] induit l'apoptose d'une lignée cellulaire T. I a mort de la lignée $T$, induite par la transfection du gène Tat, ne semble pas dépendre d'un mécanisme lié à cMyc ou Bcl-2. En revanche, la survie de la lignée T transfectée, tout comme les lymphocytes de patients infectés par le VIH, dépend des facteurs de croissance du milieu de culture. L'expression de Tat par les cellules épithéliales est sans conséquence. L'ajout de la protéine Tat recombinante à des cellules mononucléées du sang périphérique de donneurs séronégatifs induit la mort des lymphocytes $T$ mais pas celle des monocytes. L'induction d'apoptose dans la lignée T est corrélée à une activation importante des Cdk (cyclin-dependent kinases) [2]. Tat, relarguée par les cellules infectées, pourrait activer les Cdk des cellules non infectées. L'activation inappropriée des Cdk par Tat est en accord avec le fait que les lymphocytes non infectés sont anormale- ment activés chez les patients séropositifs. Ia stimulation antigénique de ces cellules non quiescentes pourrait entraîner leur mort par apoptose in vivo et la déplétion progressive des lymphocytes.

[1. Sin et M, et al. médecine/sciences $1993 ; 9$ : 1342-51.]

[2. Li C.], et al. Science 1995 ; 268 : 429-31.]

Derturbation du transport axonal dans un modèle de souris transgénique de la sclérose latérale amyotrophique. I'accumulation anormale des neurofilaments dans les motoneurones représente une caractéristique pathologique de la sclérose latérale amyotrophique (SI.A) $\left(m / s n^{\circ} 1, v o l .11, p .137\right)$. Cependant, l'importance des neurofilaments dans la maladie n'avait jamais été démontrée. Grâce à l'analyse de souris transgéniques qui surexpriment un gène humain des neurofilaments [1], Julien et son équipe de l'université McGill à Montréal (Québec, Canada) rapportent dans un récent numéro de Nature [2] que les neurofilaments peuvent contribuer à la mort des cellules nerveuses par leur accumulation excessive dans les motoneurones qui entraînerait une réduction du transport axonal de plusieurs protéines de structure et d'organites. Notamment, le nombre de mitochondries dans les fibres nerveuses est réduit, un phénomène qui entraînerait des anomalies du métabolisme énergétique de la cellule. Les motoneurones constituent une classe de cellules particulièrement vulnérables à cause de leur teneur élevée en neurofilaments. Les souris modèles de la SLA permettront de tester de nouvelles approches thérapeutiques visant à diminuer les niveaux de production des neurofilaments dans les cellules nerveuses.

[1. Côté F, et al. Cell 1993; 73: 3546.]

[2. Collard JF, et al. Nature 1995; 375: 61-4.]
Activation séquentielle des récepteurs $T$ par un complexe peptide/CMH. Les récepteurs de l'antigène des lymphocytes $T$ (TcR) reconnaissent des peptides antigéniques présentés par des molécules du complexe majeur d'histocompatibilité (CMH) [1]. L'interaction entre le TcR et le complexe peptide/CMH est faible, et il semble que des lymphocytes $T$ puissent être activés par des cellules présentant un très petit nombre de tels complexes spécifiques. Valitutti et al., de l'Institut d'Immunologie de Bâle (Suisse) [2], démontrent que ces complexes peptide/CMH peuvent recruter successivement un grand nombre de molécules TcR. Leur expérience consiste à mettre en contact des cellules présentatrices d'antigènes et des lymphocytes $\mathrm{T}$, et de calculer le pourcentage de disparition des complexes TcR/CD3 de la surface lymphocytaire lorsque la cellule présentatrice d'antigènes est chargée avec différentes concentrations de peptides spécifiques. En effet, l'interaction du récepteur $\mathrm{T}$ avec l'antigène est suivie de son endocytose, le faisant disparaître de la surface membranaire. De ces expériences, les auteurs concluent qu'un seul peptide présenté par une molécule du CMH est capable d'interagir séquentiellement avec environ 200 récepteurs. La faible affinité de l'interaction est ici un avantage puisqu'elle permet ces associations/dissociations rapides. Un tel mécanisme d'amplification de la réponse $T$ explique la très grande sensibilité de l'activation lymphocytaire par un très petit nombre d'épitopes antigéniques.

[1. Rabourdin-Combe C, el al. médecine/sciences $1991 ; 7$ : 674-80.]

[2. Valitutti S, et al. Nature 1995 ; 375: 148-51.] 